\title{
Influence of fasting, insulin and glucose on ghrelin in the dorsal vagal complex in rats
}

\author{
Lei Huang, Bin Qiu, Lin Yuan, Lili Zheng, Qiang Li and Shigong Zhu \\ Department of Physiology and Pathophysiology, Peking University Health Science Center, Beijing 100191, People's Republic of China \\ (Correspondence should be addressed to S Zhu; Email: sgzhu@bjmu.edu.cn)
}

\begin{abstract}
The dorsal vagal complex (DVC) is an important site in which ghrelin plays an orexigenic role. However, the relationship between ghrelin expression in DVC and the energy status of the organism is unclear, as well as the role of the vagus nerve in this process. In this study, ghrelin expression in DVC neurons of rats was detected, then levels of ghrelin expression were observed under the conditions of regular diet, fasting, high blood glucose, low blood glucose, and following subdiaphragmatic vagotomy and vagus nerve electrostimulation. The results showed the following: 1) there was positive staining of ghrelin neurons in DVC; 2) ghrelin protein and mRNA levels in DVC increased under fasting condition; 3) Hyperglycemia, induced by glucose production, decreased DVC ghrelin levels
\end{abstract}

and levels did not increase under hypoglycemia induced by insulin injection; 4) the dorsal trunk of the subdiaphragmatic vagus transmits a stimulatory signal to increase DVC ghrelin levels, whereas the ventral trunk transmits inhibitory information; and 5) DVC ghrelin levels decreased with $20 \mathrm{~Hz}$ stimulation on the ventral or dorsal trunk of subdiaphragmatic vagus nerves but increased with $1 \mathrm{~Hz}$ stimulation on the dorsal trunk. These results indicate that endogenous ghrelin is synthesized in DVC neurons. Conditions such as fasting, hyperglycemia, and hypoglycemia result in changes in DVC ghrelin levels in which the dorsal and ventral trunks of subdiaphragmatic vagus play different modulation roles.

Journal of Endocrinology (2011) 211, 257-262

\section{Introduction}

Ghrelin is a 28 -amino acid peptide originally extracted from rat stomach, which has been identified as an endogenous ligand for the GH secretagogue receptor (GHSR; Kojima et al. 1999). Regulation of food intake is one of the major physiological functions of ghrelin (van der Lely et al. 2004). To date, ghrelin is the only known peripheral orexigenic hormone (Kojima et al. 1999, Tschop et al. 2000, Wren et al. 2001) that plays a central orexigenic role in the hypothalamic arcuate nucleus (ARC; Cowley et al. 2003).

GHSR is extensively distributed throughout the brain. The density of GHSR is especially high in the ARC and dorsal vagal complex (DVC), including the nucleus of the solitary tract (NTS) and the dorsal motor nucleus of the vagus (DMNV; Zigman et al. 2006). It is strongly suggested that the DVC is another important site where ghrelin plays an orexigenic role. Indeed, ghrelin injected into the DVC has been shown to significantly increase food intake (Faulconbridge et al. 2003). However, previous studies focused on the biological functions of ghrelin in the DVC by its exogenous administration. So far, there is no evidence in the literature to explain the effect of endogenous ghrelin in the DVC and the relationship between ghrelin in the DVC and energy conditions, such as fasting, hyperglycemia, and hypoglycemia.
It has been reported that the vagus nerve is an essential pathway that transmits peripheral ghrelin information to the brain stem (Berthoud \& Neuhuber 2000, Date et al. 2002). It is necessary to clarify the effect of the vagus nerve on ghrelin levels in the DVC.

In this study, the expression of endogenous ghrelin in the DVC was detected by immunohistochemistry, western blotting, and quantitative PCR. The changes in DVC ghrelin levels were examined under the conditions of regular diet, fasting, and different blood glucose levels. The effect of the vagus nerve on DVC ghrelin levels was also investigated by electrostimulation or ablation of the subdiaphragmatic vagal trunk.

\section{Materials and Methods}

\section{Animals}

Eight-week-old Sprague-Dawley (SD) male rats (250-350 g) were used in this study. The rats were housed in standard plastic rodent cages and maintained in a regulated environment $\left(25^{\circ} \mathrm{C}, 12 \mathrm{~h}\right.$ light: $12 \mathrm{~h}$ darkness cycle with lights on at $0700 \mathrm{~h}$ ). Animals were fed standard rodent chow pellets with water made available ad libitum. All animal protocols were approved by the Animal Care and Use Committee of Peking University. 


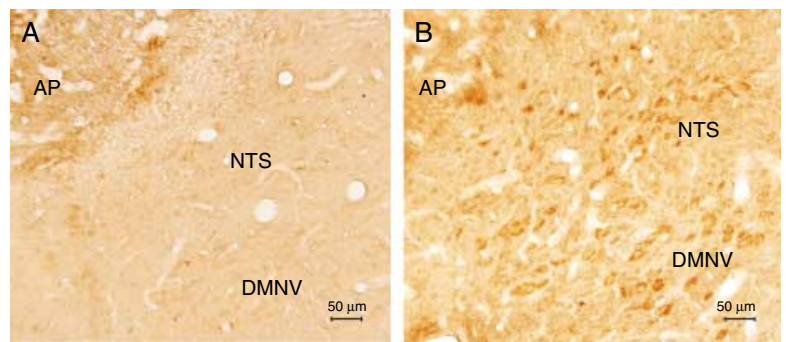

Figure 1 Photographs showing immunohistochemical localization of ghrelin in the DVC of rat. (A) Negative control staining for PBS. (B) Ghrelin-positive neurons are localized in the nucleus of the solitary tract (NTS) and dorsal motor nucleus of the vagus (DMNV) respectively. $\mathrm{AP}$, area postrema. Full colour version of this figure available via http://dx.doi.org/10.1530/JOE-11-0147.

\section{Immunohistochemistry}

Forty-eight hours before perfusion, SD rats were injected with colchicine $(200 \mu \mathrm{g}$ per rat) in the lateral ventricles to enhance the immunostaining of ghrelin-expressing neurons (Toshinai et al. 2003). Following anesthesia with an i.p. injection of sodium pentobarbital, rats were perfused with $100 \mathrm{ml} 0.9 \%$ saline through the ascending aorta for $10 \mathrm{~min}$ and then with $500 \mathrm{ml}$ fixative (4\% paraformaldehyde in $0 \cdot 1 \mathrm{M}$ PBS, $\mathrm{pH} \mathrm{7 \cdot 2)}$ for $40 \mathrm{~min}$. The brain was removed immediately and post-fixed in fixative for $4 \mathrm{~h}$ at $4{ }^{\circ} \mathrm{C}$. The brain stems were blocked, frozen at $-23^{\circ} \mathrm{C}$, and $20 \mu \mathrm{m}$ thick sections (Leica cryostat model CM1850, Wetzlar, Germany) were collected in PBS. After a number of PBS washes, the sections were incubated first in PBS containing 10\% normal rabbit serum (Zhongshan Goldenbridge Biotechnology Co., Beijing, China) and $0 \cdot 3 \%$ Triton X-100 for $30 \mathrm{~min}$ at room temperature to reduce background staining. The sections were then incubated for $18 \mathrm{~h}$ with goat anti-ghrelin antiserum (SC-10368, Santa Cruz Biotechnology, Inc., Santa Cruz, CA, USA, final dilution $1: 800)$ at $4{ }^{\circ} \mathrm{C}$ and visualized by the avidin-biotin complex (ABC) method (sc-2023, ABC kit, Santa Cruz Biotechnology, Inc.) using 0.02\% 3,3'-diaminobenzidine tetrahydrochloride (Sigma) and $0 \cdot 005 \%$ hydrogen

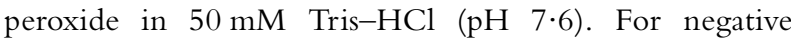
controls, the primary antibody was replaced with PBS. Photomicrographs were produced with microscope and camera (Leica DM4000B with DFC295).

\section{Animal experiments}

Fasting experiment Rats were divided into three groups (ten rats per group) and subjected to experimental fasting for 0,24 , and $48 \mathrm{~h}$. Samples from half of the animals in each group were used for western blotting, whereas the remaining samples were used for mRNA analysis.

Alteration of rat blood glucose Rats were divided into three groups (five rats per group). Control rats were injected with $500 \mu \mathrm{l}$ saline subcutaneously. The hyperglycemic rats were injected with D-glucose $(0 \cdot 2 \mathrm{~g} / \mathrm{kg}$ body weight, Sigma) subcutaneously. The hypoglycemic rats were injected with insulin (2 IU/kg body weight, Wanbang BioPharma, Jiangsu, China) subcutaneously (Nakahara et al. 2010). The blood glucose levels were detected from rattail vein using Roche Accu-Chek Performa. The DVC tissues were collected from rats $90 \mathrm{~min}$ after injection. Plasma insulin contents were determined by an ELISA kit for rats (EZRMI-13K, Millipore, St Charles, MO, USA). The limit of sensitivity of insulin assay was $0 \cdot 2 \mathrm{ng} / \mathrm{ml}$.

Subdiaphragmatic vagotomy The subdiaphragmatic vagotomy (SDV) was performed as described by Smith et al. (1981). Briefly, a midline abdominal incision was made, and the dorsal and ventral branches of the vagus nerve were exposed along the subdiaphragmatic esophagus. Each branch of the nerve was tied with surgical suture at two points separated by $\sim 1 \mathrm{~cm}$ and then transected between the sutures. Sham surgeries were also done, in which the trunk of the nerve was exposed but not cut. The rats were allowed to recover for 7 days after incision. Thirty rats were divided into three groups: sham, ventral trunk SDV, and dorsal trunk SDV. Half of the rats in each group were fed with regular diet and the remaining rats were fasted for $48 \mathrm{~h}$.

\section{Electrostimulation of subdiaphragmatic vagal} nerves The dorsal and ventral subdiaphragmatic vagal nerves were isolated from the esophagus and transected at the distal points of the vagal nerve trunks. Constant voltage stimuli $(10 \mathrm{~V}, 1 \mathrm{~ms}, 1 \mathrm{~Hz}$ or $20 \mathrm{~Hz})$ were applied to the distal end of the vagus nerve trunk for $10 \mathrm{~min}$ using bipolar platinum electrode. The brain tissues were collected $2 \mathrm{~h}$ after stimulation.

\section{Preparation of DVC tissue samples}

To prepare DVC samples, rat hindbrain tissues were quickly removed after killing the rats. Tissue blocks were transversely sectioned into $1 \mathrm{~mm}$ slices at the level of the obex using a Vibratome. Five slices with DVC were collected. The DVC area was dissected using a dissecting microscope and weighted for further western blotting assay and real-time PCR assay. The average weight of total DVC tissues was about $40 \mathrm{mg}$.

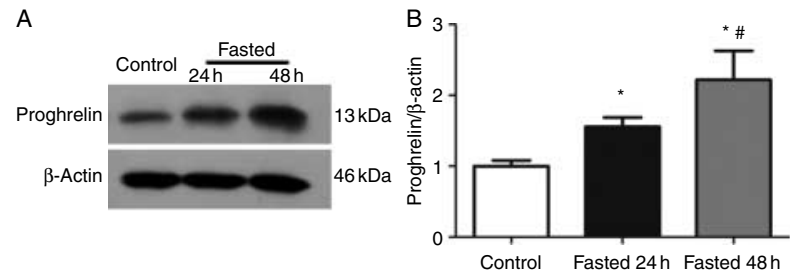

Figure 2 Fasting increased DVC ghrelin protein expression. (A) Representative western blot from fed rat (control) or rat fasted for 24 and 48 h. $\beta$-Actin was used as a loading control. (B) Quantification of image analysis of proghrelin expression is expressed as mean \pm S.E.M. ${ }^{*} P<0 \cdot 05$ vs control; ${ }^{\#} P<0 \cdot 05$ vs fasted $24 \mathrm{~h}(n=5)$. 


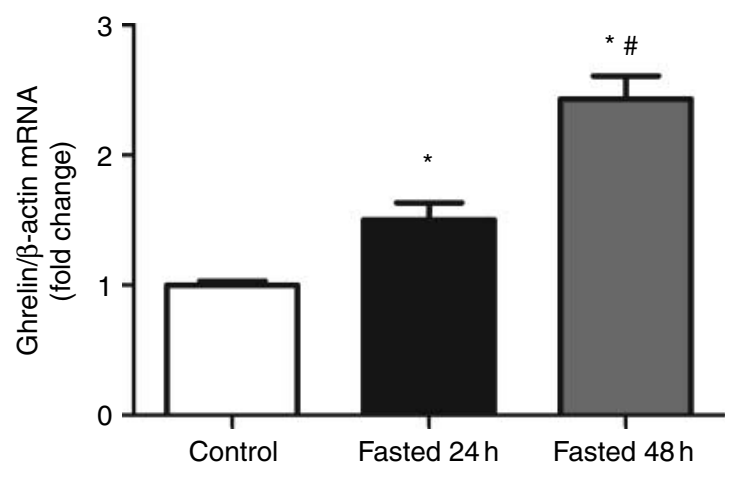

Figure 3 Fasting increased DVC ghrelin mRNA levels. Results of quantitative PCR analysis of ghrelin mRNA are expressed as fold increase under fed condition using $\beta$-actin as loading control. ${ }^{*} P<0 \cdot 05$ vs control; ${ }^{*} P<0 \cdot 05$ vs fasted $24 \mathrm{~h}(n=5)$.

\section{Western blotting}

The collected DVC tissues were rinsed thoroughly with PBS and then homogenized in cold lysis buffer (Beyotime, Jiangsu, China) plus 1:100 volume of phenylmethylsulfonyl fluoride (Beyotime). The samples were centrifuged at $12000 \mathrm{~g}$ for $15 \mathrm{~min}$ to remove debris. Protein concentration was measured using BCA kit (\#23227, Thermo Fisher Scientific, Rockford, IL, USA). A total of $80 \mu \mathrm{g}$ protein was loaded onto 15\% SDS-PAGE gel to be separated. Proteins were then transferred to nitrocellulose membrane and it was then blocked with TBS containing 5\% nonfat milk (NFM; Cat\# 232100, BD, Franklin Lakes, NJ, USA) for $1 \mathrm{~h}$. The blot was incubated with goat anti-ghrelin (sc-10368, Santa Cruz Biotechnology, Inc.; diluted $1 / 1000$, in TBS containing 5\% NFM $/ 0 \cdot 05 \%$ Tween) and rabbit anti- $\beta$-actin (1:3000; Cell Signalling Technology, Beverly, MA, USA) at $4{ }^{\circ} \mathrm{C}$ overnight. The blot was then washed three times with TBS and incubated at room temperature for $1 \mathrm{~h}$ using rabbit anti-goatHRP-conjugated secondary antibody (1:3000, KPL, Gaithersburg, MD, USA) or goat anti-rabbit-HRP-conjugated secondary antibody (1:5000, Beyotime) diluted in TBS/Tween. ECL western blotting reagent kits (Millipore, Billerica, MA, USA) were used for protein detection.

\section{Real-time PCR}

Total RNA from DVC tissue was isolated using the Trizol reagent. RT was performed using the RT system (RevertAid First-Strand cDNA Synthesis Kit, \#K1621, Fermentas, Glen Burnie, MD, USA) according to the manufacturer's instruction. Real-time PCR was conducted in a $25 \mu$ volume using real-time PCR kits (Maxima SYBR Green qPCR Master Mix, Fermentas) in Mx3000 multiplex quantitative PCR system (Stratagene, La Jolla, CA, USA). The primers used in this study were as follows: rat ghrelin, sense $5^{\prime}$-GAAGCCACCAGCTAAACTGC- $3^{\prime}$ and antisense $5^{\prime}$-GCTGCTGGTACTGAGCTCCT- ${ }^{\prime}$; and $\beta$-actin, sense
5'-ATCATGTTTGAGACCTTCAACA-3' and antisense 5'-CATCTCTTGCTCGAAGTCCA-3'. Following amplification, PCR products were analyzed by melting curve to confirm amplification specificity. Amplicon size and reaction specificity were confirmed by agarose gel electrophoresis.

\section{Statistical analysis}

All values are expressed as mean \pm s.E.M. Statistical differences were evaluated by one-way ANOVA and NewmanStudent-Keuls test. $P$ value $<0.05$ denotes statistical significance.

\section{Results}

The expression and localization of ghrelin in DVC

Ghrelin-expressing neurons were found in the NTS and the DMNV (Fig. 1B). No positive staining was detected without anti-ghrelin antiserum (Fig. 1A).

\section{Ghrelin expression in the DVC under fasting condition}

Proghrelin protein expression in the DVC significantly increased by $0 \cdot 5$ - and $1 \cdot 2$-fold compared with that of control group (fed ad libitum) when fasted for 24 and $48 \mathrm{~h}$ respectively (Fig. 2). The degree of increase was dependent on fasting time. To determine whether the increase in ghrelin protein levels was due to in situ ghrelin synthesis, we analyzed the ghrelin mRNA levels under fasting condition. The DVC ghrelin mRNA also increased by $0 \cdot 5$ - and $1 \cdot 4$-fold under fasting condition for 24 and $48 \mathrm{~h}$, respectively, compared with normal feeding condition (Fig. 3). These results indicated that chronic energy deficiency has significantly increased the DVC ghrelin synthesis.

\section{DVC ghrelin expression was affected by blood glucose levels}

To observe the effect of blood glucose on DVC ghrelin levels, blood glucose levels were altered by s.c. injection of insulin

Table 1 Blood glucose and insulin levels in rat. Control, s.c. saline; hypoglycemic rat, s.c. insulin (2 IU/kg body weight); or hyperglycemic rat, s.c. D-glucose $(0 \cdot 2 \mathrm{~g} / \mathrm{kg}$ body weight), $90 \mathrm{~min}$ after injection. Data are mean \pm S.E.M.

\begin{tabular}{|c|c|c|c|}
\hline & Control & $\begin{array}{r}\text { Hypoglycemia } \\
\text { (insulin s.c.) }\end{array}$ & $\begin{array}{l}\text { Hyperglycemia } \\
\text { (glucose s.c.) }\end{array}$ \\
\hline $\begin{array}{l}\text { Glucose } \\
(\mathrm{mmol} / \mathrm{l})\end{array}$ & $7 \cdot 17 \pm 0 \cdot 25$ & $3 \cdot 87 \pm 0 \cdot 42^{*}$ & $10 \cdot 05 \pm 0 \cdot 64^{*}$ \\
\hline $\begin{array}{l}\text { Insulin } \\
(\mathrm{ng} / \mathrm{ml})\end{array}$ & $1 \cdot 16 \pm 0 \cdot 25$ & $6 \cdot 09 \pm 0 \cdot 48^{+}$ & $2 \cdot 62 \pm 0 \cdot 36^{*}$ \\
\hline
\end{tabular}

${ }^{*} P<0 \cdot 05$ vs control; ${ }^{+} P<0 \cdot 01$ vs control, $(n=5)$. 

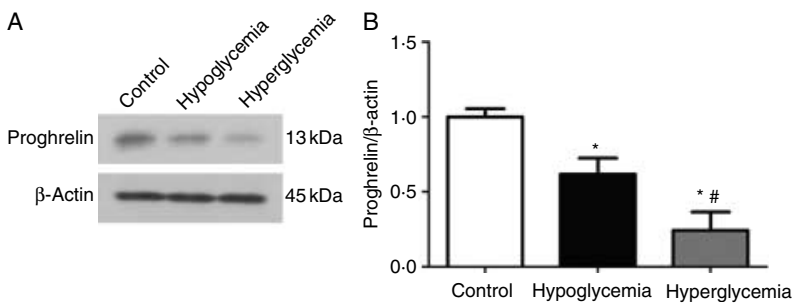

Figure 4 Effects of blood glucose level on DVC ghrelin levels. (A) Representative western blot from control (s.c. saline), hypoglycemic rat (s.c. insulin, $2 \mathrm{IU} / \mathrm{kg}$ body weight), or hyperglycemic rat (s.c. D-glucose, $0 \cdot 2 \mathrm{~g} / \mathrm{kg}$ body weight). $\beta$-Actin was used as a loading control. (B) Quantification of image analysis of proghrelin expression is expressed as mean \pm S.E.M. ${ }^{*} P<0.05$ vs control; ${ }^{\#} P<0 \cdot 05$ vs hypoglycemic rats $(n=5)$.

or glucose. The contents of insulin and glucose in blood samples were then measured, and the DVC ghrelin expression was analyzed by western blotting. Compared with saline control (Table 1 ), the blood glucose levels were significantly higher after glucose injection $(P<0 \cdot 05, n=5)$ and lower after insulin injection $(P<0 \cdot 05, n=5)$. The blood insulin contents were significantly higher than in the control group after injection with either glucose or insulin $(P<0 \cdot 05, n=5$; $P<0 \cdot 01, n=5$ respectively; Table 1$)$. Compared with saline control, DVC ghrelin expression decreased by about $75 \%$ in hyperglycemic group. However, the ghrelin expression in DVC was not increased in the hypoglycemic group; it was about $40 \%$ less than the control group (Fig. 4A and B).

\section{Effects of SDV on DVC ghrelin levels}

Fasting increased DVC ghrelin levels in intact subdiaphragmatic vagus. After ventral trunk SDV, the DVC ghrelin levels increased (Fig. 5) in both fed and fasted rats. After dorsal trunk SDV, the DVC ghrelin levels decreased under fasting or normal feeding conditions (Fig. 6). These results indicate that the dorsal trunk of the subdiaphragmatic vagus transmits a stimulatory signal to increase DVC ghrelin levels, but the ventral trunk transmits inhibitory information.

A

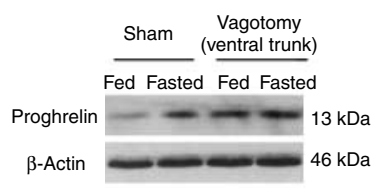

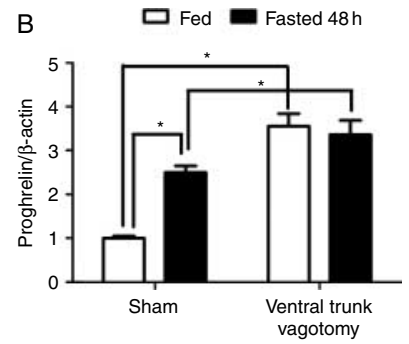

Figure 5 Effect of subdiaphragmatic vagotomy (SDV) of the ventral trunk on DVC ghrelin expression. (A) Representative western blot of rats with or without ventral trunk SDV (fed or fasted for $48 \mathrm{~h}$ ). $\beta$-Actin was used as a loading control. (B) Quantification of image analysis of proghrelin expression is expressed as mean \pm s.E.M. $* P<0 \cdot 05(n=5)$.
A
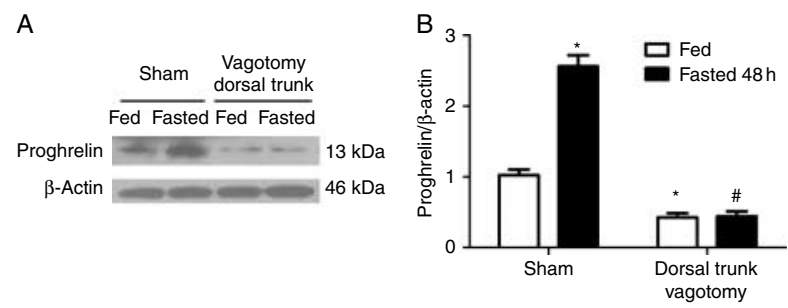

Figure 6 Effect of SDV of the dorsal trunk on DVC ghrelin expression. (A) Representative western blot from rats with or without dorsal trunk SDV (fed or fasted for $48 \mathrm{~h}$ ). $\beta$-Actin was used as a loading control. (B) Quantification of image analysis of proghrelin expression is expressed as mean \pm S.E.M. ${ }^{*} P<0.05$ vs sham (fed); ${ }^{*} P<0 \cdot 05$ vs sham (fasted for $48 \mathrm{~h} ; n=5$ ).

\section{Effects of electrostimulation of subdiaphragmatic vagus nerve on DVC ghrelin levels}

With $20 \mathrm{~Hz}$ electrostimulation on the ventral trunk of the subdiaphragmatic vagus nerve, the DVC ghrelin levels decreased by $66 \%$. The DVC ghrelin levels were not significantly changed in the group which experienced $1 \mathrm{~Hz}$ electrostimulation, compared with that of the control group (Fig. 7). In the dorsal trunk of the subdiaphragmatic vagus nerve group, DVC ghrelin levels increased by $53 \%$ under the $1 \mathrm{~Hz}$ electrostimulation and decreased by $47 \%$ under the $20 \mathrm{~Hz}$ electrostimulation (Fig. 8).

\section{Discussion}

In this study, we describe the distribution of the ghrelinpositive neurons in the NTS and the DMVN in rat DVC using immunohistochemistry. The expression of ghrelin in the DVC was confirmed by PCR and western blot. This result provides a foundation to study the relationship between the endogenous ghrelin in the DVC and the energy states of the body. We found that ghrelin protein and mRNA levels increased under fasting condition, which implies that the low blood glucose (hypoglycemia) induced by fasting may be an important factor that affects ghrelin expression in the DVC. In other words, DVC ghrelin synthesis and expression reflects the energy state of the body. To confirm this hypothesis, rats were made hyperglycemic with glucose injections and both blood glucose concentration and DVC ghrelin expression were synchronously tested. The expression of ghrelin in the DVC decreased in hyperglycemic rats, indicating that a positive energy balance inhibits DVC ghrelin levels. DVC ghrelin expression was analyzed in rats with hypoglycemia, induced by insulin injection. The rats had lower blood glucose and higher blood insulin, and the ghrelin expression in the DVC did not increase as expected; it was still lower than the saline control. This result implies that both blood glucose and insulin affect ghrelin expression in the DVC. Exogenously administered insulin rapidly increases blood insulin and exerts its effect faster than low blood glucose, which is induced by 

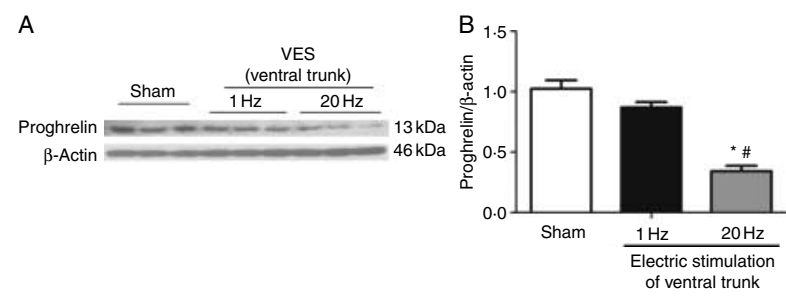

Figure 7 Effect of electrostimulation of the ventral subdiaphragmatic vagus nerve on DVC ghrelin levels. (A) Representative western blot from fed rat with sham operation or ventral subdiaphragmatic vagal electrostimulation with frequency of 1 or $20 \mathrm{~Hz}$. $\beta$-Actin was used as a loading control. (B) Quantification of image analysis of proghrelin expression is expressed as mean \pm S.E.M. ${ }^{*} P<0.05$ vs sham; ${ }^{\sharp} P<0 \cdot 05$ vs $1 \mathrm{~Hz}(n=5)$. VES, vagal electrostimulation.

insulin. Otherwise, administration of insulin is able to inhibit ghrelin secretion according to the literature (Saad et al. 2002, Flanagan et al. 2003, Iwakura et al. 2010). Therefore, it is believed that low blood glucose will induce a higher expression of ghrelin in DVC without hyperinsulinemia, for example in fasting. Moreover, one study showed that insulin receptors exist on ghrelin-producing cells and insulin inhibits ghrelin secretion in these cells (Iwakura et al. 2010). So, we speculate that insulin inhibits DVC ghrelin expression via a direct pathway, because insulin can cross the blood-brain barrier selectively (Banks et al. 1997) and the brain stem expresses insulin receptor (Unger et al. 1991). The relationship and mechanism between insulin and DVC ghrelin needs further study.

Because ghrelin receptor exists in both the DMNV and the NTS in the DVC, ghrelin may regulate the activity of the gastrointestinal system and transmit information to the highly integrated center through the DMNV and the NTS. It seems that the DVC is an essential target region for ghrelin action. Since the hypothalamus is the higher center for feeding, we speculate that the DVC is probably the preliminary center for ghrelin to control food intake. Further studies are necessary to understand the role of ghrelin expression in the preliminary center.

Since the vagus nerve is the major neuroanatomic linkage between the alimentary tract and the DVC of brain stem, the effect of vagus on ghrelin expression in DVC was examined in

A
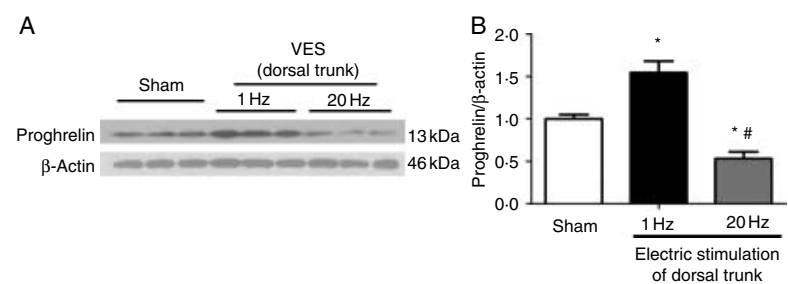

Figure 8 Effect of electrostimulation of the dorsal subdiaphragmatic vagus nerve on DVC ghrelin levels. (A) Representative western blot from fed rat with sham operation or ventral subdiaphragmatic dorsal electrostimulation with frequency of 1 or $20 \mathrm{~Hz}$. $\beta$-Actin were used as a loading control. (B) Quantification of image analysis of proghrelin expression is expressed as mean \pm s.E.M. ${ }^{*} P<0.05$ vs sham, ${ }^{\sharp} P<0 \cdot 05$ vs $1 \mathrm{~Hz}(n=5)$. VES, vagal electrostimulation. this study. The results showed that the dorsal trunk of the subdiaphragmatic vagus transmits a stimulatory signal to increase DVC ghrelin levels, but the ventral trunk transmits inhibitory information. We also found that the effect of SDV of the ventral trunk on DVC ghrelin expression is different from that of SDV of the dorsal trunk. Ablation of the ventral trunk leads to increased DVC ghrelin levels under fed condition. In contrast, ablation of the dorsal trunk leads to decreased DVC ghrelin levels. The discrepancy between the roles of the ventral and the dorsal trunks on DVC ghrelin expression may be due to their different innervations. The ventral trunk includes the common hepatic branches while the dorsal trunk does not. Moreover, the common hepatic branches take part in the transmission of blood glucose signal to brain (Lutz et al. 1996, Pocai et al. 2005). The ventral trunk may transmit signals regarding nutrient fluctuation. It has been reported that the dorsal trunk of the subdiaphragmatic vagus nerve, and not the ventral trunk, was necessary to excite locus coeruleus neurons in response to i.p. lipopolysaccharide (Borsody \& Weiss 2005) and abundant adrenergic fibers were regularly found in the peripheral stump of the splanchnic branch of the dorsal vagus trunk (Itina \& Lapsha 1985). The data suggest that the dorsal and ventral trunks may transmit different information to the brain. Further study is needed to clarify this.

Vagal afferent fibers transmit continuous spontaneous discharge that is modulated by sensory inputs (Date et al. 2002). We investigated the effect of electrostimulation of the subdiaphragmatic vagus nerve on DVC ghrelin levels. DVC ghrelin levels decreased under high-frequency stimulation of both the ventral and dorsal trunk, whilst increases in DVC ghrelin levels were observed under low-frequency stimulation of the dorsal trunk only. Grundy et al. (1995) estimate that the basic discharge of the afferent vagal was about $1 \cdot 3 \mathrm{~Hz}$ and of the distended stomach about $9 \mathrm{~Hz}$. Krolczyk et al. (2001) reported that electrostimulation with different frequencies induced vagal activity similar to the pattern observed in fed animals. So, low-frequency electrostimulation on the dorsal trunk results in stimulatory signals, similar to normal condition. While high-frequency stimulation with $20 \mathrm{~Hz}$ possibly carries a 'message' that the stomach is full and initiates the feeling of satiety.

In summary, we reveal for the first time that ghrelinproducing neurons exist in the DVC and ghrelin levels are affected by energy conditions such as fasting, insulin levels, and blood glucose levels and also by the discharge frequency of subdiaphragmatic vagus nerves. Moreover, the ventral and dorsal trunks of the subdiaphragmatic vagus nerve play opposite roles in modulating DVC ghrelin levels. The present results suggest that DVC ghrelin is involved in the regulation of energy balance.

\section{Declaration of interest}

The authors declare that there is no conflict of interest that could be perceived as prejudicing the impartiality of the research reported.

Journal of Endocrinology (2011) 211, 257-262 


\section{Funding}

This work was supported by grants from the National Natural Science Foundation of China (No. 30770804 and No. 30871034) and National Basic Science Foundation for Talents Training (No. J0630853/J0108).

\section{References}

Banks WA, Jaspan JB \& Kastin AJ 1997 Selective, physiological transport of insulin across the blood-brain barrier: novel demonstration by speciesspecific radioimmunoassays. Peptides 18 1257-1262. (doi:10.1016/S01969781(97)00198-8)

Berthoud HR \& Neuhuber WL 2000 Functional and chemical anatomy of the afferent vagal system. Autonomic Neuroscience 85 1-17. (doi:10.1016/ S1566-0702(00)00215-0)

Borsody MK \& Weiss JM 2005 The subdiaphragmatic vagus nerves mediate activation of locus coeruleus neurons by peripherally administered microbial substances. Neuroscience 131 235-245. (doi:10.1016/j.neuroscience.2004.09.061)

Cowley MA, Smith RG, Diano S, Tschop M, Pronchuk N, Grove KL, Strasburger CJ, Bidlingmaier M, Esterman M, Heiman ML et al. 2003 The distribution and mechanism of action of ghrelin in the CNS demonstrates a novel hypothalamic circuit regulating energy homeostasis. Neuron 37 649-661. (doi:10.1016/S0896-6273(03)00063-1)

Date Y, Murakami N, Toshinai K, Matsukura S, Niijima A, Matsuo H, Kangawa K \& Nakazato M 2002 The role of the gastric afferent vagal nerve in ghrelin-induced feeding and growth hormone secretion in rats. Gastroenterology 123 1120-1128. (doi:10.1053/gast.2002.35954)

Faulconbridge LF, Cummings DE, Kaplan JM \& Grill HJ 2003 Hyperphagic effects of brainstem ghrelin administration. Diabetes $\mathbf{5 2}$ 2260-2265. (doi:10. 2337/diabetes.52.9.2260)

Flanagan DE, Evans ML, Monsod TP, Rife F, Heptulla RA, Tamborlane WV \& Sherwin RS 2003 The influence of insulin on circulating ghrelin. American Journal of Physiology. Endocrinology and Metabolism 284 E313-E316. (doi:10.1152/ajpendo.00569.2001)

Grundy D, Bagaev V \& Hillsley K 1995 Inhibition of gastric mechanoreceptor discharge by cholecystokinin in the rat. American Journal of Physiology 268 G355-G360.

Itina LV \& Lapsha VI 1985 Electrophysiologic and histochemical characteristics of the fiber composition of the subdiaphragmatic portion of the vagus nerves. Fiziologicheskii Zhurnal SSSR Imeni I. M. Sechenova 71 1200-1206.

Iwakura H, Li Y, Ariyasu H, Hosoda H, Kanamoto N, Bando M, Yamada G, Hosoda K, Nakao K, Kangawa K et al. 2010 Establishment of a novel ghrelin-producing cell line. Endocrinology 151 2940-2945. (doi:10.1210/en. 2010-0090)

Kojima M, Hosoda H, Date Y, Nakazato M, Matsuo H \& Kangawa K 1999 Ghrelin is a growth-hormone-releasing acylated peptide from stomach. Nature 402 656-660. (doi:10.1038/45230)
Krolczyk G, Zurowski D, Sobocki J, Slowiaczek MP, Laskiewicz J, Matyja A, Zaraska K, Zaraska W \& Thor PJ 2001 Effects of continuous microchip (MC) vagal neuromodulation on gastrointestinal function in rats. Journal of Physiology and Pharmacology 52 705-715.

van der Lely AJ, Tschop M, Heiman ML \& Ghigo E 2004 Biological, physiological, pathophysiological, and pharmacological aspects of ghrelin. Endocrine Reviews 25 426-457. (doi:10.1210/er.2002-0029)

Lutz TA, Niijima A \& Scharrer E 1996 Intraportal infusion of 2,5-anhydro-Dmannitol increases afferent activity in the common hepatic vagus branch. Journal of the Autonomic Nervous System 61 204-208. (doi:10.1016/S01651838(96)00079-3)

Nakahara K, Okame R, Katayama T, Miyazato M, Kangawa K \& Murakami N 2010 Nutritional and environmental factors affecting plasma ghrelin and leptin levels in rats. Journal of Endocrinology 207 95-103. (doi:10.1677/JOE10-0062)

Pocai A, Obici S, Schwartz GJ \& Rossetti L 2005 A brain-liver circuit regulates glucose homeostasis. Cell Metabolism 1 53-61. (doi:10.1016/j. cmet.2004.11.001)

Saad MF, Bernaba B, Hwu CM, Jinagouda S, Fahmi S, Kogosov E \& Boyadjian R 2002 Insulin regulates plasma ghrelin concentration. Journal of Clinical Endocrinology and Metabolism 87 3997-4000. (doi:10.1210/jc.87.8. 3997)

Smith GP, Jerome C \& Gibbs J 1981 Abdominal vagotomy does not block the satiety effect of bombesin in the rat. Peptides 2 409-411. (doi:10.1016/ S0196-9781(81)80096-4)

Toshinai K, Date Y, Murakami N, Shimada M, Mondal MS, Shimbara T, Guan JL, Wang QP, Funahashi H, Sakurai T et al. 2003 Ghrelin-induced food intake is mediated via the orexin pathway. Endocrinology 144 1506-1512. (doi:10.1210/en.2002-220788)

Tschop M, Smiley DL \& Heiman ML 2000 Ghrelin induces adiposity in rodents. Nature 407 908-913. (doi:10.1038/35038090)

Unger JW, Moss AM \& Livingston JN 1991 Immunohistochemical localization of insulin receptors and phosphotyrosine in the brainstem of the adult rat. Neuroscience 42 853-861. (doi:10.1016/0306-4522(91) 90049-T)

Wren AM, Small CJ, Abbott CR, Dhillo WS, Seal LJ, Cohen MA, Batterham RL, Taheri S, Stanley SA, Ghatei MA et al. 2001 Ghrelin causes hyperphagia and obesity in rats. Diabetes 50 2540-2547. (doi:10.2337/ diabetes.50.11.2540)

Zigman JM, Jones JE, Lee CE, Saper CB \& Elmquist JK 2006 Expression of ghrelin receptor mRNA in the rat and the mouse brain. Journal of Comparative Neurology 494 528-548. (doi:10.1002/cne.20823)

Received in final form 16 August 2011

Accepted 19 September 2011

Made available online as an Accepted Preprint 19 September 2011 